\title{
Ferritin levels in critically ill patients with COVID-19: A marker of outcome?
}

\author{
George Dimopoulos ${ }^{1}$, Alexandra Sakelliou ${ }^{1}$, Aikaterini Flevari ${ }^{1}$, Kimon Tzannis ${ }^{1}$, Evangelos J. Giamarellos-Bourboulis ${ }^{2}$
}

\section{Dear Editor,}

The severe form of Coronavirus Disease 2019 (COVID-19) is a systemic disease associated with high mortality rate ${ }^{1,2}$. Elderly, mainly men with comorbidities, are at increased risk of death. Nevertheless, younger individuals, without underlying diseases, may also develop lethal complications (myocarditis, disseminated intravascular coagulopathy, neurological complications etc. $)^{3,4}$.

In the ICU of ATTIKON University Hospital (one of the 5 Reference Hospitals for COVID-19 in Athens, Greece), from 5 August to 30 September 2020, $16(100 \%)$ critically ill patients with COVID-19 were admitted (median age 70.5 years, IQR 58-79). The patients were divided into survivors [Group A: 9 (56.3\%)] and non-survivors [Group B: 7 (43.7\%)](Table1). At the time of ICU admission, the viral load of coronavirus (expressed in Circles trough: $\mathrm{Ct}$ ) was significantly higher in non-survivors [Group A: 23 (IQR 21-25) vs Group B: 21 (IQR 20-22), $p=0.042$ ], while ferritin levels were similar in both groups [Group A: 1290 ng/mL (IQR 550-3572) vs Group B: 980 (IQR 543-3915), p=0.71]. During ICU stay, the viral load remained permanently high in non-survivors [Group A: 32 (IQR 32-37) vs Group B: 22 (IQR 19-24), p=0.001], but it was gradually diminished among survivors [Group A: $39.1 \%$ (IQR 30.4-42.9) vs Group B: O (IQR -4.8-14.30), p=0.001]. In parallel, ferritin levels were increased by $109.7 \%$ (IQR 25.7-382), whatever was higher in non-survivors [Group A: 55.7\% (IQR 13.3-85) vs Group B: 486.1\% (IQR 137.2-761.9), p=0.007] (Table 1). The HScore, which is an indicator of macrophage activation, was higher in non-survivors [Group A: 54 (IQR 19-70) vs Group B: 87 (IQR 68-99), $\mathrm{p}=0.048)$ ]. Finally, in this cohort, 9 (56.3\%) patients survived and $7(43.7 \%)$ died because of ARDS/Multiple Organ Failure (MOF) (one of the patients developed myocarditis).

A consistent proportion of COVID-19 patients will develop acute respiratory distress syndrome (ARDS) related to increased production of cytokines (the so-called cytokine storm) and a small subset secondary haemophagocytic lymphohistiocytosis (sHLH), a T-cell driven hyperinflammatory, "hyperferritinemic syndrome's. These are the two main causes of mortality in the severe form of COVID-19. The sHLH development reflects the ability of coronavirus to bind TLRs and to activate inflammasome through IL-1 $\beta$ release, but the relationship is not clear since many COVID-19 patients, even with bad prognosis, do not meet the classification criteria of HScore (Table 2) $)^{6,7}$. In light of the absence of highly increased HScore, ferritin remains high and reveals constant macrophage activation albeit not to such an extent as to be the full-blown sHLH ${ }^{8-10}$. In our cohort, high viral load and ferritin levels have been observed in non-survivors indicating a relation between the activity of the disease and the outcome of the patients. A future research perspective could be focused on the following three questions: a) 'Is COVID-19 a hyperferritinemic syndrome without being full-blown sHLH?'; b) 'Is there a need to revalidate sHLH and HScore cut-off limits in these patients?'; and c) 'When in the course of the COVID-19 infection may the clinicians consider starting immunomodulatory treatment?'.

\author{
AFFILIATION \\ 1 2nd Department of Critical Care Medicine, \\ National and Kapodistrian University \\ of Athens, School of Medicine, Attikon \\ University General Hospital, Athens, Greece \\ 2 4th Department of Internal Medicine, \\ National and Kapodistrian University \\ of Athens, School of Medicine, Attikon \\ University General Hospital, Athens, Greece
}

\section{CORRESPONDENCE TO}

George Dimopoulos. 2nd Department of Critical Care Medicine, National and Kapodistrian University of Athens, School of Medicine, Attikon University General Hospital,

1 Rimini Street, 12462, Athens, Greece.

E-mail: gdimop@med.uoa.gr

\section{KEYWORDS}

COVID-19, ICU, hyperinflammation, ferritin, outcome

Received: 19 November 2020

Accepted: 24 November 2020 
Figure 1.

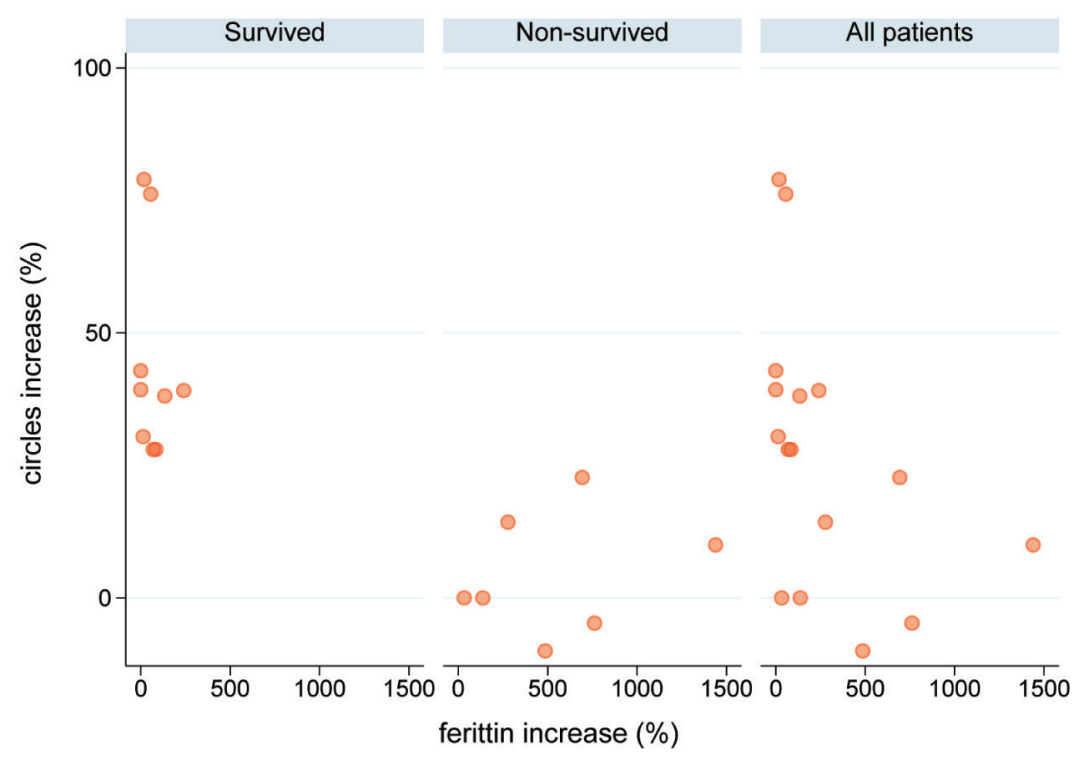

Figure 2.

Figure 3.

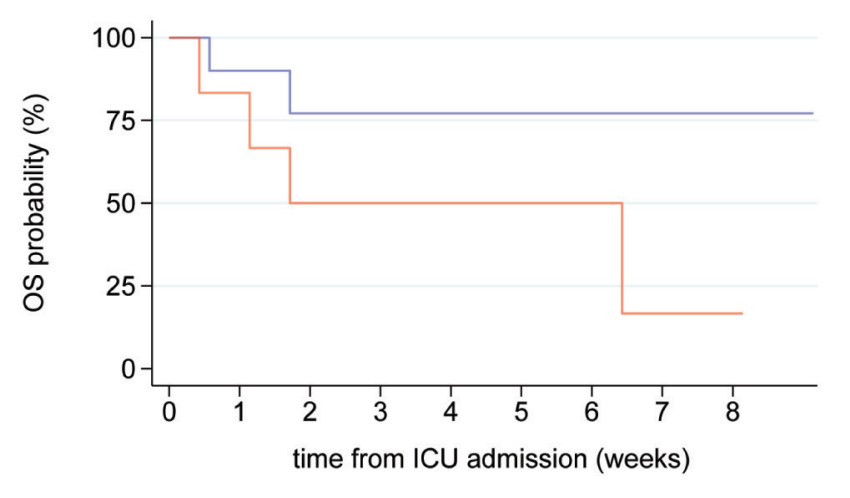

Number at risk

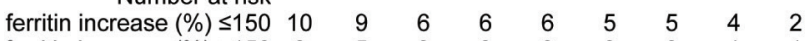

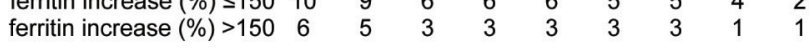
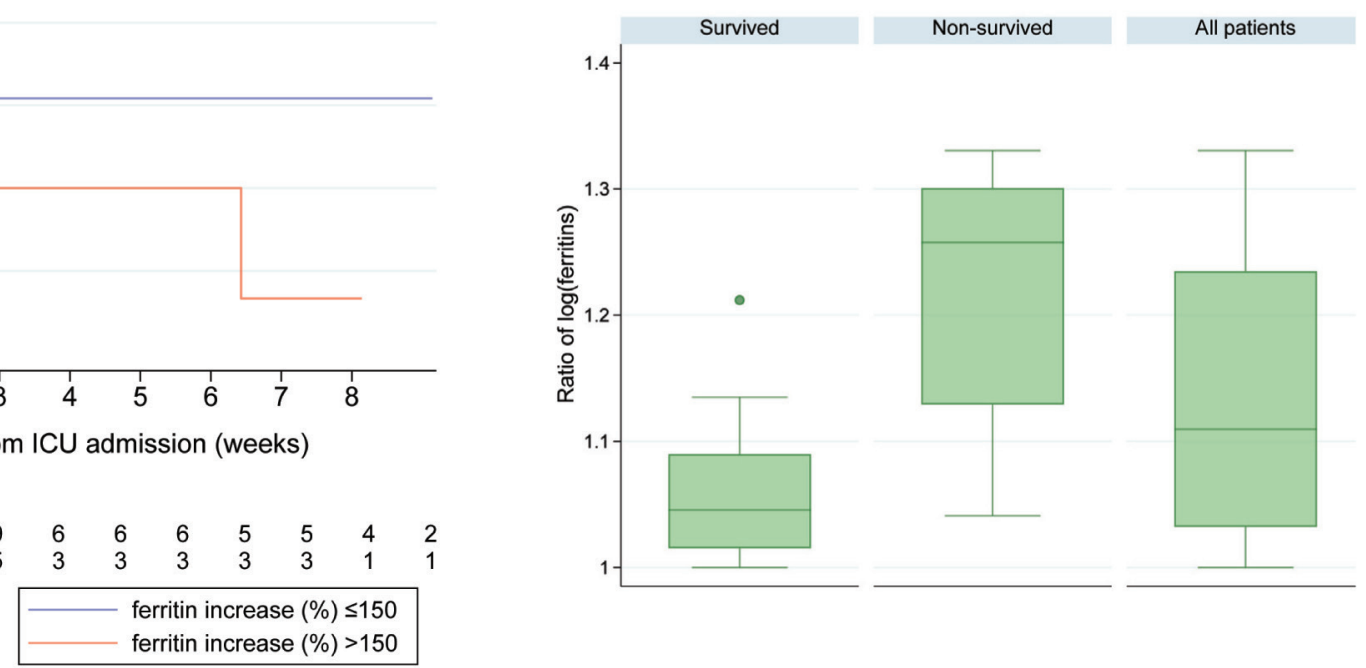

Table 1. Demographics, clinical and laboratory data of all patients, survivors and non-survivors $(\mathrm{N}=16)$

\begin{tabular}{|c|c|c|c|c|}
\hline Parameters & All patients & $\begin{array}{c}\text { Survivors } \\
\mathbf{N}=9(56.3 \%)\end{array}$ & $\begin{array}{l}\text { Non-survivors } \\
N=7(43.7 \%)\end{array}$ & $\mathbf{p}$ \\
\hline Age (years) & $70.5(58-79)$ & $75(54-79)$ & $67(62-79)$ & 0.92 \\
\hline Gender & & & & 0.55 \\
\hline Male & $13(81.3)$ & $8(88.9)$ & $5(71.4)$ & \\
\hline Female & $3(18.7)$ & $1(11.1)$ & $2(28.6)$ & \\
\hline \multicolumn{5}{|l|}{ Comorbidities } \\
\hline Hypertension & $12(8)$ & $6(66.7)$ & $6(85.7)$ & 0.59 \\
\hline Cardiovascular disorders & $7(43.8)$ & $6(66.7)$ & $1(14.3)$ & 0.06 \\
\hline Diabetes ॥ & $4(3)$ & $1(11.1)$ & $3(42.9)$ & 0.26 \\
\hline COPD & $4(25)$ & $2(22.2)$ & $2(28.6)$ & $>0.99$ \\
\hline
\end{tabular}


Table 1. Continued

\begin{tabular}{|c|c|c|c|c|}
\hline Parameters & All patients & $\begin{array}{l}\text { Survivors } \\
N=9(56.3 \%)\end{array}$ & $\begin{array}{l}\text { Non-survivors } \\
\mathrm{N}=7(43.7 \%)\end{array}$ & $\mathbf{p}$ \\
\hline Malignancy & $3(18.8)$ & $1(11.1)$ & $2(28.6)$ & 0.55 \\
\hline Epilepsy & $2(12.5)$ & $2(22.2)$ & $\mathrm{O}(0)$ & 0.48 \\
\hline Obesity & $1(6.3)$ & $1(11.1)$ & $\mathrm{O}(0)$ & $>0.99$ \\
\hline Renal failure & $1(6.3)$ & $\mathrm{O}(0)$ & $1(14.3)$ & 0.44 \\
\hline Thrombophilia & $1(6.3)$ & $1(11.1)$ & $\mathrm{O}(0)$ & $>0.99$ \\
\hline Admission & & & & 0.63 \\
\hline Directly from ER & $6(37.5)$ & $4(44.4)$ & $2(28.6)$ & \\
\hline From clinical floor & $10(62.5)$ & $5(55.6)$ & $5(71.4)$ & \\
\hline Time to ICU admission (days) & $3.5(0-7)$ & $1(0-7)$ & $4(0-5)$ & 0.87 \\
\hline APACHE II admission & $20(18.5-21.5)$ & $20(18-21)$ & $21(19-24)$ & 0.33 \\
\hline SOFA score & $13.5(11.5-15)$ & $13(12-14)$ & $15(11-15)$ & 0.23 \\
\hline Ferritin (admission), $\mathrm{ng} / \mathrm{mL}$ & 1046.5 (546.5-3748.5) & $1290(550-3572)$ & $980(543-3915)$ & 0.71 \\
\hline Ferritin (ICU stay), ng/mL & $2295(1503.5-6366.5)$ & 1735 (1289-4058) & $2538(1895-36940)$ & 0.19 \\
\hline Ferritin levels increase & $109.7(25.7-382)$ & $55.7(13.3-85)$ & $486.1(137.2-761.9)$ & $0.007^{*}$ \\
\hline $\begin{array}{l}\text { Time of highest ferritin levels (day } \\
\text { since admission) }\end{array}$ & $12.5(5.5-23.5)$ & $10(5-23)$ & $16(6-24)$ & 0.75 \\
\hline Viral load $(\mathrm{Ct})$ admission & $21.5(20.5-24)$ & $23(21-25)$ & $21(20-22)$ & $0.042^{*}$ \\
\hline Viral load $(C t)^{\mathbf{a}}$ & $29.5(22-33)$ & $32(32-37)$ & $22(19-24)$ & $0.001^{*}$ \\
\hline Viral load reduction (Ct) (\%) & $28(5-39.2)$ & $39.1(30.4-42.9)$ & $O(-4.8-14.3)$ & $0.001^{*}$ \\
\hline Temperature $\left({ }^{\circ} \mathrm{C}\right)^{\mathbf{a}}$ & $38.1(37.3-38.9)$ & $38(37.7-38.5)$ & $38.4(36-40.5)$ & 0.71 \\
\hline $\mathrm{PaO2} / \mathrm{FiO2}^{\mathrm{a}}$ & $123.5(114-159)$ & 125 (115-195) & $122(53-156)$ & 0.56 \\
\hline WBC $\left(\times 10^{9} / L\right)^{\mathbf{a}}$ & 14600 (10200-17900) & $13200(9300-15900)$ & 16900 (11980-27580) & 0.19 \\
\hline Neutrophils (\%) & $81(77.5-88)$ & $81(80-83)$ & $87(61-89)$ & 0.67 \\
\hline Neutrophils absolute number & $10380(7530-14450)$ & $10000(7460-13200)$ & $13600(7600-15100)$ & 0.32 \\
\hline Lymphocytes (\%) & $7(5-12)$ & $10(6-11)$ & $6(5-26)$ & 0.75 \\
\hline Lymphocytes absolute number & $780(695-1262)$ & $770(640-920)$ & $790(750-1290)$ & 0.47 \\
\hline$H b^{a}$ & $11.3(9.3-13)$ & $11.5(11-13)$ & $11(8.5-13)$ & 0.63 \\
\hline Platelets $\left(\times 10^{9} / \mathrm{L}\right)^{\mathbf{a}}$ & $258(224-317)$ & $252(208-318)$ & $258(249-289)$ & 0.96 \\
\hline Cytopenia $^{a}$ & $5(31.3)$ & $2(22.2)$ & $3(42.9)$ & 0.60 \\
\hline Fibrinogen $^{a}$ & $502.5(426-775.5)$ & $484(456-782)$ & $626(396-769)$ & 0.87 \\
\hline AST $(\mu K a t / L){ }^{\mathbf{a}}$ & $87(39.5-244.5)$ & $115(33-168)$ & $59(41-548)$ & 0.71 \\
\hline LDH $^{a}$ & $449.5(365-627)$ & $399(330-521)$ & $596(378-628)$ & 0.19 \\
\hline HS score ${ }^{a}$ & $69.5(50.5-87)$ & $54(19-70)$ & 87 (68-99) & $0.048^{*}$ \\
\hline Inotropic agents & $13(81.3)$ & $6(66.7)$ & $7(100)$ & 0.21 \\
\hline CRRT & $2(14.3)$ & $1(11.1)$ & $1(20)$ & $>0.99$ \\
\hline Length of ICU stay (days) & $37(8.5-52.5)$ & $52(29-57)$ & $12(4-45)$ & $0.033^{*}$ \\
\hline
\end{tabular}

Values are expressed as number (\%) or median (IQR). IQR: interquartile range. Patients presented more than one comorbidity. AST: Aspartate Aminotransferase. APACHE II: Acute Physiology And Chronic Health Evaluation II. COPD: Chronic Obstructive Pulmonary Disease. CRRT: Continuous Renal Replacement Treatment. HS score: hemophagocytic syndrome. LDH: Lactate Dehydrogenase. SOFA: Sequential Organ Failure Assessment. WBC: White Blood Cells count. a On day of highest ferritin levels. ${ }^{*} \mathrm{p}<0.05$ statistically significant. 
Table 2. Haemophagocytic lymphohistiocytosis (HLH)-2004 diagnostic criteria ${ }^{11}$ and HScore (HS) ${ }^{12}$

\begin{tabular}{|c|c|c|c|}
\hline HScore & & & \\
\hline Parameter & Number of points & Parameter & Number of points \\
\hline \multicolumn{2}{|l|}{ Temperature $\left({ }^{\circ} \mathrm{C}\right)$} & \multicolumn{2}{|l|}{ Fibrinogen $(\mathrm{g} / \mathrm{L})$} \\
\hline$<38.4$ & 0 & $>2.5$ & 0 \\
\hline $38.4-39.4$ & 33 & $\leq 2.5$ & 30 \\
\hline$>39.4$ & 49 & Ferritin $(\mathrm{ng} / \mathrm{mL})$ & \\
\hline \multicolumn{2}{|l|}{ Organomegaly } & $<2.000$ & 0 \\
\hline None & 0 & $2.000-6.000$ & 35 \\
\hline Hepatomegaly or splenomegaly & 23 & $>6.000$ & 50 \\
\hline Hepatomegaly and splenomegaly & 38 & \multirow{2}{*}{\multicolumn{2}{|c|}{$\begin{array}{l}\text { Serum Aspartate aminotransferase } \\
(I \cup / L)\end{array}$}} \\
\hline \multicolumn{2}{|l|}{ Number of cytopenias } & & \\
\hline One lineage & 0 & $<30$ & 0 \\
\hline Two lineages & 24 & $>30$ & 19 \\
\hline Three lineages & 34 & \multicolumn{2}{|l|}{$\begin{array}{l}\text { Haemophagocytosis on bone } \\
\text { marrow aspirate }\end{array}$} \\
\hline \multicolumn{2}{|l|}{ Triglycerides $(\mathrm{mmol} / \mathrm{L})$} & No & 0 \\
\hline$<1.5$ & 0 & Yes & 35 \\
\hline $1.5-4.0$ & 44 & \multicolumn{2}{|l|}{ Known immunosuppression } \\
\hline \multirow[t]{2}{*}{$>4.0$} & 64 & No & 0 \\
\hline & & Yes & 18 \\
\hline
\end{tabular}

A. A molecular diagnosis consistent with $\mathrm{HLH}$

B. Al least five of the following criteria should be met

1. Fever

2. Splenomegaly

3. Cytopenia (affecting $\geq 2$ or 3 lineages in the peripheral blood

Haemoglobin $<90 \mathrm{~g} / \mathrm{L}$ (in infants $<4$ weeks haemoglobin $<100 \mathrm{~g} / \mathrm{L}$ )

Platelets $<100 \times 10 \% / L$

Neutrophils $<1.0 \times 10^{9} / \mathrm{L}$

4. Hypertriglyceridaemia and / or hypofibrinogenaemia

Fasting triglycerides $\geq 3.0 \mathrm{mmol} / \mathrm{L}$

Fibrinogen $\leq 1.5 \mathrm{~g} / \mathrm{L}$

5. Hemophagocytosis in bone marrow or spleen or lymh nodes. No evidence of malignancy

6. Low or no NK cell activity (according to local laboratory reference)

7. Ferritin $\geq 500 \mathrm{mg} / \mathrm{L}$

8. $\mathrm{sCD} 25 \geq 2400 \mathrm{U} / \mathrm{mL}$

HScore: HS >169 is $93 \%$ sensitive and $86 \%$ specific for HLH. NK: natural killer. SCD25: soluble CD25.

\section{CONFLICTS OF INTEREST}

The authors have completed and submitted the ICMJE Form for Disclosure of Potential Conflicts of Interest and none was reported.

\section{FUNDING}

There was no source of funding for this research.

\section{ETHICAL APPROVAL AND INFORMED} CONSENT

Ethics approval and informed consent were not required for this study.

\section{PROVENANCE AND PEER REVIEW}

Not commissioned; internally peer reviewed. 


\section{REFERENCES}

1. Zhu N, Zhang D, Wang W, et al. A Novel Coronavirus from Patients with Pneumonia in China, 2019. N Engl J Med. 2020;382(8):727-733. doi:10.1056/NEJMoa2001017

2. Antoniou K, Bolaki M, Bibaki E, et al. COVID19 alert: Do we know our enemy? Pneumon. 2020;33(1):25-27. Accessed November 19, 2020. http://pneumon.org/assets/files/789/ file774_670.pdf

3. Li T, Lu H, Zhang W. Clinical observation and management of COVID-19 patients. Emerg Microbes Infect. 2020;9(1):687690. doi:10.1080/22221751.2020.1741327

4. Rello J, Belliato M, Dimopoulos MA, et al. Update in COVID-19 in the intensive care unit from the 2020 HELLENIC Athens International symposium. Anaesth Crit Care Pain Med. 2020;39(6):723-730. doi:10.1016/j.accpm.2020.10.008

5. Alunno A, Carubbi F, Rodríguez-Carrio J. Storm, typhoon, cyclone or hurricane in patients with COVID-19? Beware of the same storm that has a different origin. RMD Open. 2020;6(1):e001295. doi:10.1136/rmdopen-2020-001295

6. Ruan Q, Yang K, Wang W, Jiang L, Song J. Clinical predictors of mortality due to COVID-19 based on an analysis of data of 150 patients from Wuhan, China. Intensive Care Med. 2020;46(5):846-848. doi:10.1007/s00134-020-05991-x

7. Leverenz DL, Tarrant TK. Is the HScore useful in COVID-19? Lancet. 2020;395(10236):e83. doi:10.1016/S0140-6736(20)31057-6

8. Mehta P, McAuley DF, Brown M, et al. COVID-19: consider cytokine storm syndromes and immunosuppression. Lancet. 2020;395(10229):1033-1034. doi:10.1016/SO140-6736(20)30628-0

9. Loscocco GG. Secondary hemophagocytic lymphohistiocytosis, HScore and COVID-19. Int J Hematol. 2020;112(1):125-126. doi:10.1007/s12185-020-02895-w

10. Zhou F, Yu T, Du R, et al. Clinical course and risk factors for mortality of adult inpatients with COVID-19 in Wuhan, China: a retrospective cohort study. Lancet. 2020;395(10229):10541062. doi:10.1016/S0140-6736(20)30566-3

11. Henter JI, Horne A, Aricó M, et al. HLH-2004: Diagnostic and therapeutic guidelines for hemophagocytic lymphohistiocytosis. Pediatr Blood Cancer. 2007;48(2):124131. doi:10.1002/pbc.21039

12. Fardet L, Galicier L, Lambotte O, et al. Development and validation of the HScore, a score for the diagnosis of reactive hemophagocytic syndrome. Arthritis Rheumatol. 2014;66(9):2613-2620. doi:10.1002/art.38690 